\title{
Die religiöse Identität von Frauen im Spannungsfeld von Zugehörigkeit, Autorität und Autonomie ${ }^{1}$
}

\author{
Angelika Walser
}

Taking some snapshots from everyday religious life in Austria as a starting point, my contribution is a theoretical reflection on women's religious identity in the area of tension between belonging, authority and autonomy. Following postmodern concepts of identity I describe women's religious identity as "threshold identity" resp. as fluid and fragile. This description requires a clarification of the relation between secular and religious identity on one side and religious and moral identity on the other. It becomes evident that the experience of God as a constituent of religious identity exceeds by far the moral identity of the religious self, comprising a special potential of emancipation for women. However, realizing this potential in the sense of empowerment and personal autonomy results in conflicts with male authorities, who as the guardians of religious traditions, have a key function in the construction and demarcation of religious identity. If women's threshold identity goes along with a substantial concept of autonomy, entailing the call for institutional gender justice, it is the challenge of our time in all religious traditions, especially in Christianity and Islam. Here is the crucial point to decide what the religious landscape of the future will look like and whether women of the 21 st century will find a home in their religious traditions.

Religious identity; womens' identity; moral identity; womens' autonomy; religion and gender; womens' emancipation and religious authorities

Angelika Walser is Professor and Chair in Moral Theology at Salzburg University. Her research activities focus on: Feminist ethics and gender, bioethics (especially artificial reproductive technologies), spiritual theology, interreligious dialogue, literature and theology. Recent publications: In deiner Nähe geht es mir gut. Warum Freundschaften lebensnotwendig sind (Innsbruck 2017); Ein Kind um jeden Preis? Unerfüllter Kinderwunsch und künstliche Befruchtung. Eine Orientierung (Innsbruck 2014).

\section{Die religiöse Identität von Frauen - Momentaufnahmen}

Die religiöse Identität von Frauen bewegt sich im Spannungsfeld von Zugehörigkeit, Autorität und Autonomie. Damit ist bereits angedeutet, dass eine genaue Lokalisierung dieser Identität schwer fällt. Der folgende Beitrag versucht es

1 Ich danke meiner Kollegin Sigrid Rettenbacher für ihre hilfreichen Kommentare zu meinen Gedanken. 
dennoch und beginnt an der Oberfläche: Zwei Momentaufnahmen heutiger Realität bieten ein scheinbar klares Bild (1.1.). Doch die Bilder sind in Bewegung geraten und die Suche nach einem genauen Ort der religiösen Identität von Frauen erweist sich als komplexe Tiefenbohrung (1.2.) ...

\subsection{Betrachtung zweier Fotos und ein Bericht aus der Praxis der Lehre}

Die folgenden beiden Fotos dokumentieren eine Tatsache, die zumindest für Frauen - ob sie sich selbst als ,religiös“ oder als „,nicht religiös“ definieren - wenig überraschend ist: Bei einem Gipfeltreffen von Vertretern religiöser Traditionen (in diesem Fall katholische, orthodoxe und koptische Kirchenoberhäupter) beherrschen im Großen und Ganzen Männer das Bild. ${ }^{2}$ Ihnen ist die Repräsentanz von Religion in der Öffentlichkeit vorbehalten. Sie sitzen an den Schalthebeln der Macht und in den Leitungsfunktionen und tragen damit die offizielle Letztverantwortung. ${ }^{3}$

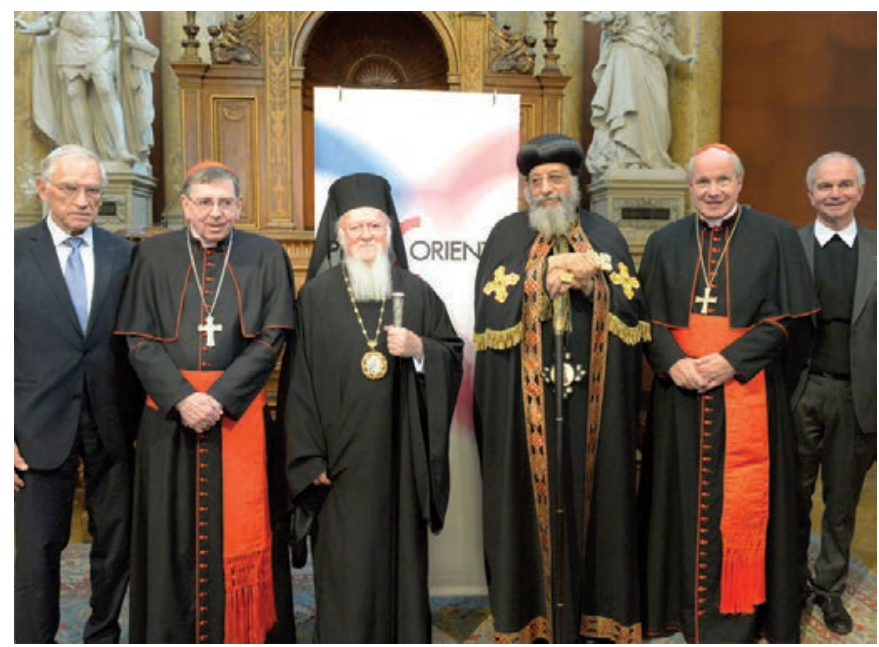

Bild 1: Die Repräsentation und die Leitungsverantwortung in der Öffentlichkeit den männlichen Autoritäten ... ${ }^{4}$

2 Wie immer bestätigen Ausnahmen - vor allem im protestantischen Christentum die Regel.

3 Vgl. Internetquellen: Studien des Schweizer Interreligiösen Think-Tanks 2011.

4 Vgl. Internetquelle: „Kirchen gemeinsam zum Schutz der Nahost-Christen verpflichtet". 
Zwar gerät das Bild in letzter Zeit langsam aber sicher in Bewegung: Die Appelle der internationalen ökumenischen Frauenkonferenzen bzw. des ÖRK bezüglich Geschlechtergerechtigkeit bei der Frauenordination, die bis in die Mitte des vergangenen Jahrhunderts zurückreichen und Leitgedanken feministischer Theologie in konkrete Aktionen umsetzten, ${ }^{5}$ haben zumindest in vielen protestantischen Kirchen mittlerweile zu einem langsamen aber deutlichen Strukturwandel beigetragen ${ }^{6}:$ Hier finden sich zunehmend Frauen in der Rolle der Chefin, konkret in der Rolle der Bischöfin. Obwohl die orthodoxen Kirchen als auch der Katholizismus von einem solch geänderten Bild noch weit entfernt sind, sind zumindest in der katholischen Kirche in jüngster Zeit ebenfalls vorsichtige Anzeichen eines gewissen Reflexionsprozesses wahrzunehmen: So spricht Papst Franziskus in seinem nachsynodalen Schreiben "Amoris Laetitia“ von der gesellschaftlichen Notwendigkeit von Frauen in Entscheidungspositionen (AL 54) sowie in der Priesterausbildung (AL 203). Von einem Nachdenken über die Frauenordination für das Priesteramt will Papst Franziskus explizit absehen. ${ }^{7}$ Wohl wird aber derzeit die Möglichkeit der Einführung des Diakonats für Frauen in der katholischen Kirche überprüft. ${ }^{8}$

Ein verändertes Bild zeigt sich neuerdings auch zumindest für den Islam in Westeuropa, der ebenfalls zunehmend um ein weibliches Gesicht in der Öffentlichkeit und um die Bestellung von Chefinnen bemüht ist, um auf diese Weise antiislamischen Tendenzen entgegenzusteuern. ${ }^{9}$

Man darf mit Recht die kritische Rückfrage stellen, ob allein schon die Repräsentation einer Religion durch eine Frau in der Öffentlichkeit als Ausdruck einer besseren PR-Strategie die institutionellen Machtgefüge selbst wirklich wesentlich transformieren wird. Eine vereinzelte Alibifrau in dieser und jener Führungsfunktion macht bekanntlich noch keinen strukturellen Wandel aus. Bisher jedenfalls muss man daher - wenn man über die religiöse Identität von Frauen sprechen möchte - von einem anderen Bild ausgehen:

5 Eine ausführliche Darstellung findet sich bei Meyer-Wilmes 1990, S. 19-41.

6 Vgl. Internetquelle: Lutheranworld.

7 Seit der mehrfach bestätigten Erklärung der Glaubenskongregation „Inter insigniores“ im Jahr 1976 hält die katholische Kirche an diesem strikten Nein zur Frauenpriesterweihe fest.

8 Vgl. Internetquelle: Frauen als Diakone.

9 So liegt die Leitung der Islamisch-Religionspädagogischen Akademie Wien (IRPA) in den Händen einer Frau. Auch die IGGÖ, die Islamische Glaubensgemeinschaft in Österreich, setzt vermehrt auf Frauen in Leitungsfunktionen, vgl. Internetquelle: IRPA. 


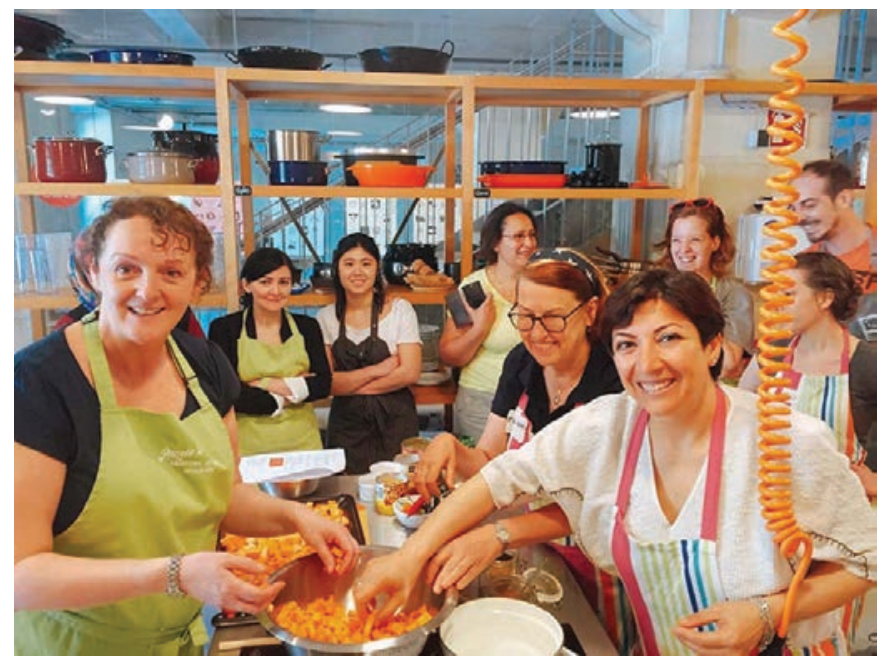

Bild 2: ... das zivilgesellschaftliche und praktische Engagement den Frauen ${ }^{10}$

Treten Religionen bzw. Konfessionen als zivilgesellschaftliche Akteure auf die Bühne der Öffentlichkeit, wird die Szene von Frauen dominiert: Ihr caritatives gesellschaftliches Engagement ist ungebrochen hoch. Sie sind in der Flüchtlingsarbeit vertreten, sorgen für Alte, Kranke und Kinder und beziehen einen wesentlichen Teil ihres Selbstbewusstseins aus dieser typisch weiblichen Fürsorgearbeit für andere. Frauen, die sich zu einer bestimmten religiösen Tradition als ,zugehörig“ definieren, haben in dieser Rolle der Fürsorgerin einen festen und akzeptierten, durch die Tradition gesicherten Ort. Dies gilt im Übrigen nicht nur für Religionen und Konfessionen, sondern aufgrund der klassischen Trennung von Erwerbs- und Fürsorgearbeit auch für die gesamte Zivilgesellschaft.

Deutlich mehr Schwierigkeiten beim Versuch einer genauen Ortsbestimmung der religiösen Identität von Frauen zeigt eine zweite Momentaufnahme aus der Praxis, diesmal entnommen der Lehre an einer Hochschule. Als Professorin unterrichtete ich nicht nur katholische und evangelische, sondern auch orthodoxe Studentinnen, allesamt angehende Religionslehrerinnen in Volks- und Hauptschulen in Österreich. Im Rahmen der Seminare schilderten sie mir übereinstimmend eine Erfahrung, die ich im Folgenden als Ausgangspunkt meines Nachdenkens zum Anlass nehmen möchte, nämlich eine auffallende Ambivalenz bezüglich der Zugehörigkeit zur eigenen konfessionellen Gemeinschaft: „Ich gehöre dazu und ich gehöre gleichzeitig auch nicht dazu. Ich gehöre dazu, wenn wir miteinander in der Kirche beten und singen. Da bin ich Teil meiner Gemeinschaft. Wenn es aber darum geht, mit wem ich schlafe, wann ich ein Baby

10 Vgl. Internetquelle: Caritas Wien. 
Die religiöse Identität von Frauen im Spannungsfeld

bekommen will, wann ich heiraten will oder ob ich gar nicht heiraten will - das entscheide ich selbst. Die Vorgaben meiner Kirche sind mir da relativ egal.“

Andeutungen in Begegnungen mit muslimischen Studentinnen im Rahmen einer Kooperation zwischen meiner Hochschule und der Islamisch-Religionspädagogischen Akademie (IRPA) in Wien ließen ähnliche Erfahrungen erkennen: Im Westen sozialisierte junge muslimische Frauen - angehende islamische Religionslehrerinnen - erwähnten bei aller Loyalität und Zugehörigkeit zu ihrer Religion die gleichzeitige Erfahrung der Nicht-Zugehörigkeit. Von ihr war insbesondere dort die Rede, wo Fragen der individuellen Lebens- und Familienplanung bzw. moralisch-ethische Fragen bezüglich Sexualität und Partnerwahl berührt wurden. ${ }^{11}$ Die oft nur versteckt geäußerten Andeutungen ließen den Schluss zu, dass den Studentinnen die Kluft zwischen ihrem Wunsch nach persönlicher Freiheit und Selbstbestimmung und den Vorgaben der weltweiten bzw. österreichischen islamischen Glaubensgemeinschaft durchaus bewusst war.

Eine dritte Momentaufnahme vertieft den Eindruck von Ambivalenz bezüglich Zugehörigkeit und Nichtzugehörigkeit.

\subsection{Frauen an der Schwelle zwischen profanem und sakralem Raum - eine Begegnung}

Im Jahr 2014 hatten Angehörige der feministischen Organisation „Frauenhetz“"12 in Wien, einem feministischen Forum bzw. einer Frauenbildungsstätte für feministische Kultur und Politik, protestantische, katholische und islamische Theologinnen zu einem Gedankenaustausch in kleinem Kreis eingeladen. Sie griffen damit Jürgen Habermas Plädoyer für einen doppelten Lernprozess bezüglich der Grenzen von Aufklärung und religiösen Lehren auf ${ }^{13}$ und setzten seinen Vorschlag um, in einer postsäkularen Gesellschaft religiös musikalischen Bürgern und Bürgerinnen zuzuhören und sie mit ihrem speziell religiösen Hintergrund in den Diskurs über ethische Fragen in der Gesellschaft bewusst einzubinden. Es handelte sich also um eine Konfrontation zwischen „säkularen“ Feministinnen und akademisch-theologisch hochgebildeten Frauen, wobei letztere entweder an Hochschulen lehrten oder in ihrer jeweiligen Religion/Konfession Leitungs- und Repräsentationsfunktionen für ihre jeweilige Religion ausübten.

Sehr schnell stand das Thema der Identität religiöser Frauen im Raum, denn die „säkularen Feministinnen“ formulierten die Frage nach der Bruchlinie sehr direkt: „Wie könnt Ihr als gebildete und emanzipierte Frauen des 21. Jahrhunderts der römisch-katholischen Kirche und der Umma angehören und damit

11 Genaue empirische Untersuchungen zu dieser Beobachtung sind ein Forschungsdesiderat.

12 Vgl. Internetquelle: Frauenhetz.

13 Habermas 2005, S. 115 f. 
einem patriarchal-hierarchischen System mit festzementierten und ontologisch bzw. schöpfungstheologisch begründeten Geschlechterverhältnissen? Wie verträgt sich eine solche religiöse Identität mit dem, was ureigenstes Anliegen des Feminismus in der Tradition der Aufklärung ist: Gleichberechtigung zwischen den Geschlechtern, Emanzipation der Frau? Gebt Ihr Frauen an der Schwelle zur katholischen Kirche oder zur Moschee Eure säkulare Identität auf? Lasst Ihr sie sozusagen vor den Eingangstüren stehen, um aufzugehen im großen singenden und betenden Kollektiv? Zieht Ihr diese zurückgelassene Identität nach dem religiösen Ritual wieder an - wie die staubigen Schuhe? Tarnt Ihr säkulare Werte wie Autonomie, eigenständiges Denken unter dem Schleier? Nehmt Ihr sie sozusagen inkognito an die heilige Stätte mit, verbergt sie dort aber notwendigerweise, weil es sonst zu schweren Konflikten mit den religiösen Autoritäten käme? Seid Ihr gar so etwas wie ,gewollt schizophren', könnt die religiöse Identität einund die säkulare Identität ausschalten, ganz nach Belieben und Situation?" Die Antwort der Theologinnen spiegelte das Ringen um eine klare Ortsbestimmung wider: Sie betonten ihre Loyalität zu ihrer jeweiligen religiösen Tradition und wiesen allesamt auf die großen Transformationsprozesse hin, die sich in Protestantismus, Islam und Katholizismus bezüglich des Themas der Geschlechtergerechtigkeit bereits vollzogen hätten oder gegenwärtig vollzögen; sie erzählten von internen Spannungen und Kontroversen. Doch beharrten sie darauf, ihre jeweilige Tradition als den Raum zu deuten, in dem für sie Gotteserfahrung möglich wird. Daher könne ihre Zugehörigkeit auch nicht einfach wie eine Vereinsmitgliedschaft abgelegt werden. Sie signalisiere neben religiöser auch kulturelle Heimat und Geborgenheit. Sogar der Protest gegen fehlende Geschlechtergerechtigkeit ${ }^{14}$ sei als Teil der eigenen Identität zu deuten und bewahre vor vorschnellen Vereindeutigungen. ${ }^{15}$ Er bilde das Potential zu notwendigen Veränderungen und zwar zu Veränderungen in beiden Sphären - sowohl im öffentlichen Raum als auch im Binnenraum der eigenen religiösen Tradition. Dass letztere dem Anspruch der Emanzipation bzw. auch dem Anspruch der Geschlechtergerechtigkeit einer säkularen Gesellschaft nicht genüge, gestanden alle Theologinnen zu, weshalb sie ihre eigene Position als ,Dazwischen“ beschrieben - an der Schwelle zwischen sakralem und profanem Raum. Dieses Dazwischen ist mit einer gewissen Ortlosigkeit verbunden. Die Schwelle signalisiert einen Bereich, der zwischen zwei Räumen liegt und daher mit Unsicherheit einhergeht: Ist Frau drinnen oder draußen? Gehört sie in den religiösen oder in den säkularen Raum? Ist sie überhaupt irgendwo zuhause oder sitzt sie buchstäblich zwischen allen Stühlen?

14 Für einen religionswissenschaftlich-systematischen Überblick über das Thema der Geschlechtergerechtigkeit in religiösen Traditionen, vgl. Heller 2014a, S. 29-44.

15 Vgl. Sommer 2002, S. 294-296. 


\section{Theoretische Reflexion der Praxis}

\subsection{Religiöse Identität an der Schwelle}

Frauen, die sich selbst als zu einer religiösen Tradition zugehörig definieren und gleichzeitig typisch moderne Werte wie Emanzipation und Geschlechtergerechtigkeit bejahen, leben in einer Art Grenzbereich. Sie definieren sich häufig als Grenzgängerin und werden aus der Sicht der anderen auch als solche wahrgenommen. Konzepte wie der sog. third space von Homi Bhabha können dieser Selbst- und Fremddefinition Rechnung tragen. ${ }^{16}$ Die Raummetapher macht deutlich, wie vage, fluid und fragil die eigene Identität in diesem Zwischenbereich erlebt wird. Die oben skizzierten Momentaufnahmen verdeutlichen die Ambivalenz der Erfahrung von Zugehörigkeit und Nicht-Zugehörigkeit und signalisieren, wie schwierig eine klare Selbstvergewisserung und Selbstverortung ist: „Dabei markiert der dritte Raum eine zentrale sprachphilosophische und erkenntnistheoretische Einsicht: Es gibt keine unveränderliche Bedeutung und keine fixe Form der Repräsentation. Identitätszuschreibungen sind vielmehr ein liminales Geschehen, ein Grenzereignis [...]."17 Erst der Diskurs über diese Identität schafft die angeblich klaren Grenzen zwischen Innen und Außen, lässt eine hybride Identität zu einer zumindest halbwegs gesicherten Identität werden, die im sozialen Geschehen des Alltags unumgänglich ist.

Die religiöse Identität der angehenden Theologiestudentinnen in 1.1. bzw. der Theologinnen in 1.2. kann weiter als eine vagabundierende oder nomadisierende Identität beschrieben werden - eine Identität, die nicht festgelegt ist und immer auf der Suche nach sich selbst, immer unterwegs, bleibt. ${ }^{18}$ Es ist eine fragmentarische Identität, die sich nur aus dem jeweiligen Kontext erschließt. ${ }^{19}$

Wohlgemerkt bezog sich die Beschreibung der "Schwellenidentität“ in den Momentaufnahmen des letzten Kapitels bisher auf eine kleine und herausgehobene Gruppe religiöser Frauen, nämlich auf Studentinnen und Professorinnen der Theologie bzw. Frauen, die in Führungs- und Repräsentationsfunktionen innerhalb ihrer jeweiligen religiösen Tradition eine exponierte Stellung haben. Die Frage der Zugehörigkeit oder Nichtzugehörigkeit stellt sich für sie in ganz besonderem Maße, denn die typischen Werte der westlichen Moderne wie Emanzipation und Gleichberechtigung fordern die Frage nach Loyalität und Zugehörigkeit zu einer religiösen Tradition geradezu heraus, weil sie mit den Geschlechterkonzepten diverser religiöser Traditionen - beispielsweise mit dem Konzept einer typisch weiblichen Wesensart und damit verbundenen normativen

16 Vgl. Bhabha 1994.

17 Rettenbacher 2013, S. 91.

18 Vgl. Braidotti 1993.

19 Vgl. Benhabib 1995. 
Vorgaben im katholischen Christentum und Islam - nicht ohne weiteres vereinbar sind.

So differenziert die hier beschriebenen Theologinnen von der Religionsforschung bezüglich ihrer jeweiligen persönlichen Religiosität/Spiritualität, ihrer Religion und ihrem Commitment in ihrer religiösen Gemeinschaft auch erfasst werden können, ${ }^{20}$ so sehr verbindet sie - wenn auch mit graduellen Unterschieden - der Spagat zwischen diesen Vorgaben ihrer religiösen Traditionen und dem Anspruch einer modernen Gesellschaft an emanzipierte und gleichberechtigte Frauen.

Zulehner und Steinmair-Pösel haben in ihrer Studie „Typisch Frau?“ eine wachsende Akzeptanz „moderner" Frauenbilder in Österreich erhoben und zwar nicht nur für den Bereich „Leben“, sondern auch für den Bereich „Glauben““. Hier zeigt sich, dass die Frage eines gleichberechtigten Miteinanders von Männern und Frauen für eine überwiegende Mehrzahl von Frauen - unabhängig von ihrem kirchlichen Commitment - ein neuralgischer Punkt ist: Selbst zwei Drittel der sog. „traditionsbewussten Frauen“ (66 \%) halten Gleichberechtigung für die Lösung gesellschaftlicher Probleme für wichtig. Sog. ,,moderne Frauen“ sprechen sich zu 89 \% dafür aus. Eine beeindruckende Mehrheit aller Frauen (und Männer) in Österreich votiert entsprechend der normativen Leitvorstellung von Gleichberechtigung für die Zulassung von Frauen zu den kirchlichen Ämtern, sogar die sog. ,,traditionsbewussten“ Frauen (61 \%).

Auch wenn es die religiöse Frau in der Realität nicht gibt und die soeben skizzierte Gruppe von Theologinnen keineswegs repräsentativ ist für alle evangelischen, katholischen und islamischen Frauen, kann angesichts dieser Ergebnisse von Zulehner und Pösel doch mit einiger Vorsicht - Vorsicht deshalb, weil die untersuchten Frauentypen ausschließlich geborene Österreicherinnen sind vermutet werden, dass die religiöse Identität von Frauen grundsätzlich und immer wieder als eine in Frage stehende, herausgeforderte, unsichere und fragile Identität in einem „Dazwischen“ erfahren wird - eine Identität, die Bild 2 bejaht, sich aber durch Bild 1 immer wieder herausgefordert und angefragt sieht. Eine solche

20 Ich folge hier den drei großen Dimensionen der Religionsforschung (Religiosität, Religion, Kirchlichkeit) nach Zulehner / Steinmair-Pösel 2011, S. 128.

21 Ebd., S. 19-85: „Moderne Frauen“ bejahen die Frauenemanzipation, legen Wert auf ihre Berufstätigkeit und favorisieren geteilte Erwerbs- und Fürsorgearbeit gemeinsam mit dem Partner. „Traditionsbewusste Frauen“ halten am traditionellen Modell vom Mann als „Versorger“ der Familie fest und halten Frauen grundsätzlich dafür geeigneter, sich der Hausarbeit und den Kindern zu widmen. Zwischen beiden profilierten Polen finden sich allerdings zwei weitere Typen, nämlich die „pragmatischen“ Frauen, die sozusagen aus beiden Lebenskonzepten das jeweils passende wählen und eine Gruppe von Frauen, die sich weder in den alten noch in den neuen Mustern beheimatet fühlen. Zulehner und Steinmair-Pösel haben sie die „suchenden Frauen“ genannt. 
Die religiöse Identität von Frauen im Spannungsfeld

Identität lässt sich tatsächlich nicht leicht fixieren und muss immer wieder neu diskursiv erschlossen werden.

Angesichts dieses Befunds ist die in der Kapitelüberschrift verwendete Metapher der Schwelle als nur vage markierte Grenze in einem Zwischenraum zwischen zwei Räumen durchaus legitim. Gleichzeitig bedarf sie jedoch der kritischen Reflexion: Ihre Verwendung könnte nämlich immer noch den missverständlichen Eindruck erwecken, dass säkularer und religiöser Raum und die damit jeweils verbundene religiöse und säkulare Identität klar voneinander abgegrenzt und getrennt werden könnten. Genau dies jedoch ist sehr fraglich geworden.

\subsection{Das Verhältnis zwischen säkularer und religiöser Identität}

Jürgen Habermas hat in seiner Friedenspreisrede von 2001 mit seiner Formulierung von der sog. postsäkularen Gesellschaft einen interdisziplinären Diskurs angestoßen, dessen Geschichte an dieser Stelle nicht nochmals aufgerollt werden soll. ${ }^{22}$ Die Debatte um die Bedeutung von Religion(en) im öffentlichen Raum einer Gesellschaft und den Begriff der Säkularisierung wird seit Jahren geführt und hat besonders auch auf Seiten der Religionssoziologie zu notwendigen Differenzierungen in der inhaltlichen Bestimmung dessen geführt, was Säkularisierung heute bedeutet. ${ }^{23}$ Als Katalysator der Auseinandersetzung mit der Säkularisierungsthese erwiesen sich die Studien des amerikanischen Soziologen José Casanova, welcher den europäischen Gründungsmythos vom säkularen europäischen Staat, der mithilfe der Aufklärung die irrationale und gefährliche Religion in eine geschützte private Sphäre verbannt und damit gezähmt hat, einer Kritik unterzog. Casanova konnte erstens zeigen, dass dieser europäische Gründungsmythos historisch ungenau, wenn nicht schlichtweg falsch ist; zweitens wurde durch seine Analysen deutlich, dass dieser Gründungsmythos machtpolitischen Interessen dient bzw. der Abgrenzung vom Islam, der derzeit als die große Bedrohung Europas wahrgenommen wird. Die Zugehörigkeit zu einer religiösen Tradition als ein Faktor von Identität wird zum willkommenen Vehikel der Exklusion, denn der Mythos eines aufgeklärten modernen und demokratischen Europas kann nur aufrechterhalten werden, wenn er sich vor dem Schatten des dunklen irrationalen und der Vormoderne verhafteten Fundamentalismus von Religion, insbesondere der islamischen Religion, umso strahlender abheben kann. $^{24}$

22 Habermas 2001. Einen Überblick über die Debatte bietet Heimbach-Steins 2006, S. 9-24.

23 Pollack 2014, S. 93-120.

24 Casanova 2008, S. 109. 
Feministische Politikwissenschaftlerinnen und Soziologinnen wie Joan W. Scott von der Princeton University und Niamh Reilly aus Irland haben Casanovas Thesen rezipiert: Beide widersprechen der im säkularen Feminismus lange Zeit geltenden selbstverständlichen Annahme, dass eine zunehmende Säkularisierung und Modernisierung der Gesellschaften Europas automatisch zu mehr Geschlechtergleichheit und -gerechtigkeit führe, weil ja der Hort des Beharrens auf der sexuellen Differenz - die Religion - als Relikt der Vergangenheit zurückgelassen worden sei. So schreibt Joan W. Scott in ihrem Essay „Sexularism“: „I want to argue, contrary to so many contemporary claims, not only that there is no necessary connection between [secularism and gender equality], but also that the equality that secularism promises was always - still is - troubled by sexual difference. “25 Anhand von Textanalysen der französischen und der russischen Revolution weist Scott nach, dass Geschlechtergerechtigkeit weder für die Väter der Französischen noch der Russischen Revolution irgendeine Rolle spielte. Gleichzeitig macht sie deutlich, dass in der ersten feministischen Bewegung dezidiert eine religiöse Motivation eine zentrale Rolle für den politischen Kampf um Emanzipation spielte. Die Rede von der säuberlich getrennten religiösen und säkularen Identität geht also nicht auf. Der Säkularismus - also die Ideologisierung von Säkularisierung - liefert laut Scott keine Lösungen bestehender Geschlechterungleichheit. Er bietet lediglich die willkommene Möglichkeit, die eigenen Schwierigkeiten bezüglich sexueller Differenz auf andere Kulturen und Religionen zu projizieren.

Ähnlich urteilt Niamh Reilly in ihrem Artikel „Rethinking the interplay of feminism and secularism in a neo-secular age": Auch sie ruft dazu auf, das Säkularisierungskonzept aus feministischer Perspektive neu zu überdenken, damit nicht am Ende ausgerechnet im Namen der Aufklärung wichtige Errungenschaften wie die Religionsfreiheit abgeschafft werden, nur um an einem falschen Ideal einer rationalen und modernen, weil religionsfreien Öffentlichkeit festhalten zu können. Sie kritisiert die Nichtthematisierung von Religion in feministischer Theoriebildung, die angesichts der Praxis absurd sei: „Hence, if women make up the majority of actively religious (or spiritually oriented) people, it follows, that ,feminism“ - as a conceptual and practical project concerned with the ,emancipation of women' - must acknowledge the role of religion in women's lives in more complex and nuanced ways than has happened to date. " 26

Was bedeuten diese Einsichten für die Frage nach der religiösen Identität von Frauen? Zunächst einmal, dass die Behauptung einer Dichotomie zwischen „religiös“ und „säkular“ und eine damit verbundene Identitätsvorstellung in Frage gestellt werden muss. Die Blockbildung „,religiöse Frauen“ gegen ,säkulare Frauen“, „Verschleierte“ gegen „Unverschleierte“, „Rationale“ gegen „Irrationale“, „Sklavinnen“ gegen „Emanzipierte“ geht schlicht nicht auf. Erstens ist die

\footnotetext{
25 Scott 2009, S. 3.

26 Reilly 2011, S. 19.
} 
Wirklichkeit weit komplexer, was allein schon die Tatsache belegt, dass es längst moderne Formen einer säkularen Religiosität/Spiritualität vor allem im Bereich der Alternativmedizin und im Wellnessbereich gibt, in der vor allem Frauen - unabhängig von religiöser Zugehörigkeit oder kirchlichem Commitment - Hilfe zur Bewältigung ihres Alltags finden. ${ }^{27}$ Religiosität/Spiritualität ist also keineswegs notwendigerweise auf die großen religiösen Traditionen beschränkt. Die angeblich „säkularen“ Frauen - auch die „säkularen Feministinnen“ aus der Gesprächsrunde - sind nicht rein säkular = irreligiös.

Zweitens lässt sich die Zugehörigkeit zu einer Religion als Teil des großen Puzzles oder Mosaiks begreifen, das die Identität jeder einzelnen Frau in ganz unterschiedlicher Art und Weise mitbestimmt. Hier muss ernstgenommen werden, was im Fachjargon Intersektionalität genannt wird, nämlich die Tatsache, dass Frauen (wie übrigens auch Männer) auf sehr unterschiedliche Art und Weise in ihrer eigenen Religion sozialisiert sind, wobei die Zugehörigkeit zu einer Religion immer in Wechselwirkung zu Milieu, Geschlecht, Ethnie, Alter und weiteren anderen Faktoren steht. Die säkulare Frau gibt es ebenso wenig wie die religiöse Frau. Jeder Versuch, eine solche Identität genau zu fixieren, handelt sich zu Recht den Vorwurf ein, sie stünde im Dienst eines essentialistischen Konzepts von Identität.

Identitäten sind keine fix vorgegebenen Entitäten, die man ein für alle Mal festschreiben könnte. Vielmehr sind Identitäten etwas Dynamisches - sie werden im jeweiligen Kontext diskursiv hervorgebracht, wobei die eigene Identität unter Abgrenzung von konkurrierenden Identitäten konstruiert wird. Die Definitionsmacht und Repräsentationshoheit sind dabei meist nicht gleichwertig verteilt $[\ldots]^{28}$

Postkoloniale Theorien machen darauf aufmerksam, dass Identitäten als diskursive Konstruktionen verstanden werden müssen, innerhalb derer das hervorgebracht wird, was benannt werden soll - „die Anderen“. Die Konstruktion „der Anderen" liefert Gewissheit und Selbstversicherung für die eigene Identität und funktioniert über Abgrenzung. Die eigene Identität muss sich unterscheiden, wie Christian Danz es im Anschluss an Peter L. Berger formuliert hat:

Für die Vergewisserung der eigenen Identität kommt Religionskulturen eine grundlegende Funktion zu. Durch ihr kollektives Gedächtnis beantworten sie die Frage, wer wir sind, und stiften durch ihre Narrative krisenresistente, kollektive und individuelle Identitäten mit starken normativen Gewissheiten, welche die Funktionen von Inklusion und Exklusion erfüllen. Dadurch liefern Religionskulturen klare Orientierungen und Unterscheidungen in einer unübersichtlich gewordenen Welt. ${ }^{29}$

27 Vgl. Heller 2014, S. 57 und 129.

28 Rettenbacher 2013, S. 85.

29 Danz 2013, S. 36. 
Die Konstruktion des eigenen Selbst in Abgrenzung zum Selbst „des Anderen“ ist normativ besetzt und bedient sich u. a. der moralischen Bewertung des Lebensstils ,des Anderen“. Dabei wird auf normative Vorgaben in der eigenen religiösen Tradition zurückgegriffen, mit deren Hilfe Grenzziehungen vorgenommen werden, u. a. mithilfe moralisch-ethischer Haltungen und Handlungsempfehlungen.

Es ist jedoch nicht nur die Moral, welche bei der Konstruktion des „Eigenen“ im Gegensatz zum „Anderen“ eine gewichtige Rolle spielt. Es sind auch die religiösen Autoritäten, die in allen großen religiösen Traditionen dieser Welt beanspruchen, moralisch-ethische Leitbilder an die kommende Generation weiter zu tradieren und damit in besonderem Maße Garanten der kollektiven religiösen Identität zu sein. Die meisten in der Weltöffentlichkeit agierenden Autoritäten sind insbesondere in den monotheistischen Religionen traditionellerweise männlich (siehe Bild 1). Ihre Autorität prägt in entscheidendem Maße die religiöse Identität von Frauen mit. Bevor diesem Zusammenhang und dem Begriff der Autorität im nächsten Kapitel genauer nachgegangen werden soll, ist im Hinblick auf die normativen Gewissheiten, welche der Inklusion von „Dazugehörigem“ und der Exklusion von „Nicht-Dazugehörigem“ dienen, zunächst das Verhältnis von moralischer Identität zur religiösen Identität genauer zu untersuchen.

\subsection{Das Verhältnis zwischen religiöser und moralischer Identität}

Die Schwierigkeit vieler aufgeklärter Zeitgenossen und -genossinnen im Umgang mit den großen religiösen Traditionen resultiert aus einer immer noch wirkmächtigen aber falschen Identifizierung von Moral mit Religion, wie sie seit Kant bis heute nachwirkt - unter anderem auch deshalb, weil fundamentalistische Vertreter von Religionen ihre Abgrenzung gegenüber einer angeblich bösen und teuflischen Welt anhand moralischer Kategorien vornehmen. ${ }^{30}$ Moralische Identität wird gerne und schnell mit religiöser Identität gleichgesetzt. Gegenüber einer solch unzulässigen Gleichsetzung von moralischer und religiöser Identität gilt es jedoch aus theologischer Sicht Einspruch einzulegen. ${ }^{31}$

In der theologischen Ethik christlicher Prägung sind seit langem einschlägige neutestamentliche Stellen angeführt worden, die bereits als innerbiblische Kritik an einer Verkürzung von Religion auf Moral aufzufassen sind. Am besten lässt sich dies am Beispiel des Gleichnisses vom barmherzigen Vater (Lk 15, 11-32)

30 Als Beispiel sei hier die Verurteilung von Homosexualität genannt, die beispielsweise in Teilen Afrikas nach wie vor Gang und Gäbe ist und dort von männlichen Autoritäten im Namen der Heiligen Schriften wie Koran und Bibel bzw. unter Berufung auf den Willen Gottes und im Namen einer christlichen bzw. islamischen Identität praktiziert wird.

31 Mandry hat dazu den Grundstein gelegt, vgl. Mandry 2002. 
oder auch am Beispiel der Arbeiter im Weinberg (Mt 20,1-16) verdeutlichen: In beiden Gleichnissen ist von der Barmherzigkeit Gottes die Rede, die gängige moralische Vorstellungen von Gerechtigkeit auf den Kopf stellt: Der verlorene Sohn, der als Sünder heimkehrt, wird mit einem Festmahl begrüßt; die Arbeiter im Weinberg, die als letzte angeworben wurden, erhalten genau denselben Lohn wie alle anderen, obwohl sie eine deutlich verkürzte Arbeitszeit hatten. Die Barmherzigkeit Gottes, bei der im hebräischen Wortlaut nicht umsonst die „rachamin“, die Gebärmutter anklingt, schenkt neues Leben jenseits moralischer Kategorien. Die zugesagte Vergebung ist offensichtlich unabhängig von einer moralischen Vorleistung des Menschen. Religiöse Identität, in diesem Fall jüdisch-christliche Identität, erweist sich an Stellen wie diesen explizit als moralkritisch, insofern Gottes Liebe eben gerade nicht mit Lohn und Strafe operiert, sondern mit der Zusage der Umkehrmöglichkeit:

Religion bleibt hinter Moral zurück und ist ihr doch zugleich voraus, wenn man bedenkt, dass im christlichen Glauben von der Rechtfertigung des Sünders, von der Befreiung von Schuld und von Erlösung die Rede ist. Die moralischen Fähigkeiten und Wertigkeiten des Menschen, das ist ja gerade das Befreiende an der christlichen Botschaft, machen nicht des Menschen Annahme von Gott her aus. ${ }^{32}$

Die Gotteserfahrung des religiösen Selbst, wie sie in solchen Texten über die Jahrtausende hinweg überliefert wird, übersteigt traditionelle moralische Vorstellungen bei weitem: In den genannten Gleichnissen geht es nicht um eine Art Tauschgerechtigkeit oder um die Beschwörung einer infantilen Gottesvorstellung, in welcher der oberste Herrschergott durch moralisches Wohlverhalten seiner Söhne/Töchter oder Sklaven milde gestimmt wird, sondern um die revolutionäre Einsicht, dass Gott jenseits von Lohn und Strafe Umkehr und neues Leben ermöglicht.

Eine weitere Stelle ist zu nennen, die sich in die Grundaussage der bereits genannten Gleichnisse einfügt, sie aber um die vorher angesprochene Autoritätsfrage erweitert: Es handelt sich um die Begegnung Jesu mit der Frau aus Samarien am Jakobsbrunnen (Joh 4,1-30). V. 18 hat in der Auslegungsgeschichte des Textes stets zu Spekulationen über das moralisch fragwürdige Leben der samaritanischen Frau geführt. Doch worin auch immer der Grund der rechtlich ungeordneten Beziehungen dieser Frau zu ihren Männern bestanden hat - feministische Theologie äußert die Vermutung, es könnte sich um den typischen Fall einer Leviratsehe gehandelt haben ${ }^{33}$ - so ist doch eines klar: Sie wird von Jesus nicht moralisch verurteilt. Er bittet sie um Wasser, spricht mit ihr, enthüllt sich ihr zuletzt sogar als Messias. „Die Andere“, die fremde Frau aus Samaria, die den moralischen Vorgaben ihrer Gesellschaft mitsamt ihrer Autoritäten nicht genügt,

32 Mieth 1999, S. 163 f.

33 Habermann 2007, S. 530-533. 
wird in und nach der Begegnung mit Jesus zur Autorität ihres eigenen Lebens und zu einer Autorität der neuen Gemeinde in Samaria: Sie ist es, die zu den Ihren eilt und ihnen die Botschaft vom Messias mitteilt (V. 29). Feministische Theologie hat das befreiende Potential des Glaubens an Jesus gerade für die religiöse Identität von Frauen immer erkannt und betont. Der Gott des Alten Testaments verspricht gerade den ärmsten und schwächsten Mitgliedern seines Volkes, den Witwen und Waisen, eine bessere Zukunft und umfassenden Frieden, Shalom, sowie Gerechtigkeit für alle Geschöpfe. Die großen Propheten und Prophetinnen des AT wie auch Jesus wenden sich genau den Marginalisierten zu - unabhängig von ihrer moralischen Perfektion, denn nach Mt 9,12 brauchen eben gerade nicht die Gesunden den Arzt, sondern die Kranken.

Eine Gleichsetzung zwischen moralischer Identität und religiöser Identität ist also im Blick auf solche Stellen nicht möglich. Allerdings gibt es zwischen beiden Analogien. Das moralische Selbst ist ein ansprechbares Selbst - ein Selbst, das den Anruf des/der Anderen, des Nächsten, des Mitmenschen, hören und beantworten kann. Es konstituiert sich stets in einem intersubjektiven Akt. Die Vorstellung, dass von dem oder der Anderen kraft seines/ihrer Existenz eine Art moralische Inanspruchnahme ausgeht, die im Selbst einen Widerhall findet, dass der/die Andere also eine Art Quelle ethischer Aufforderung ist, wird in allen Abhandlungen über moralische Identität entfaltet, sehr häufig unter Bezugnahme auf Paul Ricoeur und Emmanuel Levinas. ${ }^{34}$

Die Analogie zur Erfahrung des religiösen Selbst ist deutlich greifbar: Auch Gott wird in der Bibel als der dem Menschen zugewandte Gott charakterisiert, erscheint als anrufbar bzw. ruft von sich aus den Menschen an und beruft ihn. So hat feministische Theologie beispielsweise Maria als von Gott berufene Prophetin interpretiert, welche im Magnifikat (Lk 1, 46-55) die neue Gerechtigkeit Gottes gerade für die Armen und Marginalisierten verkündet. Geschlechtergerechtigkeit lässt sich im Hinblick auf solche Stellen theologisch begründen. Die Gotteserfahrung des religiösen Selbst fordert das moralische Selbst heraus, indem es darauf beharrt, dass die Erfahrung Gottes eine Befreiung hin auf mehr Zukunft, auf mehr Gerechtigkeit ist - gerade auch für Frauen. Die religiöse Identität von Frauen enthält - insofern sie eingebettet ist in den Strom einer Identitätskommunikation von Generation zu Generation, der solche Befreiungserfahrungen in seiner Erinnerung bewahrt ${ }^{35}$ - die Ahnung, dass moralisches Wohlverhalten im Sinne einer reinen Anpassung an traditionelle moralische Vorgaben noch keine Gotteserfahrung ist und dass religiöse Identität immer einen Mehrwert gegenüber moralischer Identität enthält.

Was ist bisher gewonnen? Anhand einiger Momentaufnahmen aus der Praxis wurde deutlich, dass die religiöse Identität von Frauen, die sich einer religiösen Tradition zugehörig fühlen, berechtigterweise als Schwellenidentität oder als

34 Vgl. Mandry 2002.

35 Vgl. Hervieu-Leger 2000. 
Die religiöse Identität von Frauen im Spannungsfeld

Identität im Grenzbereich beschrieben werden kann. Dabei wurde allerdings die Gefahr einer missbräuchlichen Verwendung der Metapher im Sinne eines essentialistischen Identitätsbegriffs und den damit verbundenen Exklusionstendenzen im Sinne eines gegenseitigen „Othering“ aufgezeigt, das Identitäten im Sinne eines performativen Akts erst hervorbringt. Weiter wurde betont, dass religiöse und moralische Identität nicht gleichzusetzen sind bzw. dass die Gotteserfahrung gerade Frauen dazu bringen kann, traditionelle moralische Vorgaben, für deren Durchsetzung jahrhundertelang vor allem männliche Autoritäten zuständig waren, in Frage zu stellen. Unter Berufung auf die Autorität Jesu können religiöse Frauen - wie die samaritanische Frau - neue Lebensmöglichkeiten für sich entdecken, die weit über normative Vorgaben moralischer Art hinausreichen und individuelle Neuaufbrüche ermöglichen. Hier kommen also Werte ins Spiel, die religiösen Traditionen keineswegs fremd sind, sondern inhärent: Die samaritanische Frau gewinnt durch die Begegnung mit Jesus nicht nur neues und erlösendes Wissen, sondern auch neue Autorität im Sinne von Handlungsvollmacht über ihr eigenes Leben. Ihre Gotteserfahrung bringt aber auch Bewegung in das gewohnte „Ortsbild“ der bisherigen männlichen Autoritäten. Es drängt nach außen an die Öffentlichkeit. Die Frau, welche den Messias bezeugt hat, wird zur Wegbereiterin der neuen Gemeinde von Samaria (Joh 4,42). Sie ist auch als Frau mit fragwürdigem moralischen Ruf eine religiöse Autorität für andere geworden und eine lebendige Erinnerung daran, wie Bild 1 aussehen könnte. Ihre Glaubenserfahrung lässt sich als Erfahrung von Empowerment beschreiben - als Befähigung, das eigene Leben selbst in die Hand zu nehmen. Eine solche Erfahrung aber lässt sich nicht auf das Privatleben beschränken, sie wird und muss öffentlich werden, um wirksam werden zu können.

\section{Die religiöse Identität von Frauen im Spannungsfeld von Autorität und personaler Autonomie}

Der Begriff der „Autorität“ ist in diesem Zusammenhang bislang wörtlich verstanden worden: Die samaritanische Frau wird durch die Begegnung mit dem Messias nicht nur zur „Urheberin“ und „Begründerin“ einer neuen Gemeinde, sondern auch zur Autorität ihres eigenen Lebens. ${ }^{36}$ Das Johannesevangelium erzählt nichts über das spätere Leben dieser Frau und nichts über ihr weiteres Verhältnis zu den offiziellen männlichen Autoritäten. Ein Blick in Vergangenheit und Geschichte der Theologie zeigt aber, dass die Gottesbegegnung vieler Frauen und die aus ihr resultierende Selbstautorisierung über das eigene Leben die offiziell anerkannten männlichen Autoritäten oft in Frage stellen. ${ }^{37}$ Die Tatsache,

36 „auctor“ meint: Urheber, Gründer. Vgl. Kluge 1989, S. 51.

37 Als Beispiel sei nur die Geschichte aller großen Mystikerinnen genannt. 
dass eine Frau durch ihre Gotteserfahrung deutlich „gewachsen“38 ist, stellt das Bild von den untergeordneten gehorsamen Frauen gegenüber den übergeordneten männlichen Autoritäten in Leitungsfunktionen auf den Kopf.

\subsection{Zwischen Selbstermächtigung und traditionellen Autoritäten: Religiöse Identität unter Druck}

Religiöse Identität kann unter dem Vorzeichen einer Gotteserfahrung an der Schwelle zwischen alle Fronten und unter erheblichen Druck geraten: Während die überkommenen Autoritäten der eigenen religiösen Tradition Gehorsam fordern, strebt das religiöse Selbst angesichts der erlebten Gotteserfahrung nach Selbstermächtigung und Selbstbestimmung.

Hannah Arendt hat Autorität sowohl als Eigenschaft einzelner Personen wie auch als Eigenschaft eines Amtes definiert. ${ }^{39}$ Kennzeichen der Autorität ist die „fraglose Anerkennung seitens derer, denen Gehorsam abverlangt wird; sie bedarf weder des Zwanges noch der Überredung. " ${ }^{40}$ Autorität ist nicht immer, aber häufig mit Macht verknüpft, wobei Macht

[...] der menschlichen Fähigkeit [entspricht], nicht nur zu handeln oder etwas zu tun, sondern sich mit anderen zusammenzuschließen und im Einvernehmen mit ihnen zu handeln. Über Macht verfügt niemals ein Einzelner; sie ist im Besitz einer Gruppe und bleibt nur solange existent, als die Gruppe zusammenhält. ${ }^{41}$

Beide - Autorität und Macht - sind für Arendt relationale Kategorien: Die fraglose Anerkennung der einen basiert auf dem stillschweigenden Gehorsam der anderen.

Für diese Untersuchung bedeutet das: Die Gotteserfahrung des religiösen Selbst von Frauen kann traditionelle (männliche) Autoritäten gefährden oder sogar zu Fall bringen. Statt einer teilweisen oder gänzlichen Fremdbestimmung weiterhin unreflektiert Gehorsam zu leisten, entdecken Frauen sich hier ganz neu als Autorität über das eigene Leben. Dies ist die Ausgangserfahrung jeder feministischen Theologie: Die Erfahrung der Befreiung zu sich selbst. Feministische Theologie behauptet seit jeher, dass sie vorzugsweise dort stattfindet, wo man sie zunächst gar nicht vermutet: an der Grenze, an der Schwelle, im „Dazwischen“. Oder, um es mit Paul Tillich zu sagen: „Die Grenze ist der eigentliche Ort der Erkenntnis. ${ }^{\text {"42 }}$ Die Grenze oder die Schwelle ist ein Raum, der in eigenartiger und

38 lat. augere: mehren, wachsen, vgl. Kluge 1989, S. 51.

39 Arendt 1990, S. 46.

40 Ebd., S. 46.

41 Ebd., S. 45.

42 Tillich 1962, S. 13. Zitiert nach Meyer-Wilmes 1990, S. 7. 
Die religiöse Identität von Frauen im Spannungsfeld

besonderer Weise offen scheint für Gotteserfahrung. Er ist damit auch der Raum, in dem sich die besondere Spannung zwischen Fremdbestimmung durch externe Autoritäten und Selbstbestimmung aufbaut. Doch was heißt Selbstbestimmung?

\subsection{Prozedurale und substantielle Autonomiekonzeptionen}

Selbstbestimmung oder personale Autonomie kann mit Herlinde Pauer-Studer folgendermaßen definiert werden „Freiheit bedeutet die Fähigkeit, eine autonome Person zu sein, d.h. autonom die Art und Form des Lebens, welches man führen will, zu bestimmen, sofern dies mit der gleichen Freiheit von anderen verträglich ist."43 Die personale Autonomie, die in der kulturellen Tradition westlicher Aufklärung bzw. im angelsächsisch-amerikanischen Raum beheimatet ist, ist als Selbstbestimmung im Sinne einer aktiven Umsetzung der eigenen Wünsche und persönlichen Lebenspläne übersetzbar - im Gegensatz zu Kants Konzeption, der mit Autonomie die Fähigkeit zur Sittlichkeit und die Umsetzung selbstgewählter Selbstbeschränkung durch moralische Prinzipien wie den kategorischen Imperativ versteht.

Personale Autonomie ist ein Dachbegriff für sehr unterschiedliche Konzeptionen, die - einer groben Unterscheidung folgend - prozeduraler oder substantieller Art sein können. Nur die zweite - die substantielle Konzeption enthält Visionen guten Lebens, wie sie auch in den Religionen enthalten sind.

Prozedurale Konzeptionen von personaler Autonomie untersuchen, ob ein Akteur/eine Akteurin eine selbstbestimmte Entscheidung und eine daraus resultierende Handlung getroffen hat: Zwei Kriterien sind ausschlaggebend für die Beurteilung, nämlich (1) das Kriterium der Authentizität und (2) die prozedurale Unabhängigkeit.

Das erste Kriterium, das Kriterium der Authentizität, stellt die Frage, ob es wirklich das moralische Selbst war, das entschieden und gehandelt hat? Haben seine Wünsche und Bestrebungen zu dieser oder jener Entscheidung und Handlung geführt oder sind es die Wünsche der anderen? Lebt das moralische Selbst sein Leben oder das der anderen? In der Diskussion stehen zahlreiche Verfahren zur Überprüfung dieser Frage. ${ }^{44}$ Welchen materialen Gehalt diese Entscheidungen/Handlungen haben, ist in der Version der prozeduralen Autonomie nicht relevant. Gerald Dworkin, Vertreter eines prozeduralen Autonomiebegriffs kommt in seiner Studie zum Autonomiebegriff zur Schlussfolgerung: „Let me conclude by pointing out that, on my view, there is no specific content to the decisions an autonomous person may take. An autonomous person may be a saint or sinner, a rugged individualist or a conformist, a leader or a follower. “45

43 Pauer-Studer 2000, S. 15.

44 Für einen Überblick über die verschiedenen Prüfverfahren vgl. Dworkin 1989.

45 Dworkin 1988, S. 62. 
Das zweite Kriterium der prozeduralen Unabhängigkeit untersucht die inneren und äußeren Bedingungen, wie eine Entscheidung/Handlung zustande kommt: Bestand Wahlfreiheit und Handlungsfreiheit bzw. Willensfreiheit oder lagen Zwänge innerer und äußerer Art vor? Eine Person, die unter äußeren Zwängen (beispielsweise Gefangenschaft) oder inneren Zwängen (beispielsweise Wut, Drogen oder Hypnose) handelt, von anderen manipuliert, getäuscht oder betrogen wird, hat nur eine eingeschränkte oder gar keine Freiheit und damit nur sehr wenige oder vielleicht gar keine Handlungsoptionen. Die Testfrage lautet hier jeweils im Nachhinein: „Hätte ich unter diesen Umständen auch anders entscheiden und handeln können?“ Wenn diese Frage mit „Ja“ beantwortet werden kann, ist der Lackmus-Test für Autonomie grundsätzlich positiv verlaufen.

Feministische Philosophinnen wie Oshana haben diesen prozeduralen Weg kritisiert. ${ }^{46}$ Er sei zu individualistisch und bedenke nicht in ausreichendem Maße die soziale Konstruktivität des moralischen Selbst und damit die Tatsache, dass gesellschaftliche Normen gerade von Frauen so stark internalisiert werden, dass eine Handlung nach außen zwar als autonom erscheint, es in Wahrheit aber nicht ist. Interessanterweise werden im Diskurs hier sehr oft religiöse Frauen als Beispiel angeführt: So argumentieren feministische Vertreterinnen US-amerikanischer Moralphilosophie, dass weder das Leben einer Muslima noch das Leben von Frauen, die evangelikalen Kirchen angehören, als autonom bezeichnet werden können, weil die hierarchischen und autoritären sozialen Strukturen dieser religiösen Traditionen die Autonomie von Frauen von vornherein verhinderten. Eine freie Entscheidung gegen das Ideal der Selbstbestimmung könne nicht Ausdruck von Selbstbestimmung sein, weil hier der Wert der Selbstbestimmung selbst verletzt und traditionelle Sichtweisen von der untergeordneten Rolle der Frau unterstützt würden. ${ }^{47}$ Personale Autonomie müsse daher stark substantiell gefasst werden. Ein rein prozeduraler Zugang sei naiv und reiche nicht aus, weil er dem Faktor der prägenden Gemeinschaft nicht Rechnung trage.

Religiöse Frauen, vor allem traditionell lebende Muslima, haben gegen diese Sichtweise verständlicherweise protestiert, wird ihnen hier doch die Fähigkeit zur personalen Autonomie einfach abgesprochen. Tatsächlich muss im politisch-öffentlichen Diskurs und daher auch im interreligiösen Dialog sehr genau überlegt werden, ob mit einem prozeduralen oder einem substantiellen Autonomiebegriff operiert wird. Der prozedurale Weg hat den Vorteil, dass er sich eines Urteils über individuelle Lebensformen enthält und damit auch religiösen Frauen - hier insbesondere auch traditionell lebenden religiösen Frauen - die Möglichkeit einer

46 Vgl. Nedelsky 1989, S. 25; Oshana 2006, S. 81. Die feministische Diskussion über den Autonomiebegriff wird in der bisher unveröffentlichten Habilitationsschrift von Angelika Walser beschrieben, welche an der Universitätsbibliothek Wien zugänglich ist: Walser 2013.

47 Vgl. Oshana 2015, S. 3-24 bzw. Warriner 2015, S. $25-47$. 
autonomen Lebensführung grundsätzlich einmal zugesteht. ${ }^{48}$ Sie alle können sich als respektierte Mitglieder mit ihren Meinungen und Entscheidungen in gesellschaftliche und politische Prozesse einbringen. Politisch gesehen sind daher prozedurale Autonomiekonzeptionen für einen interreligiösen Dialog zwischen religiösen Frauen gut geeignet. Sie sprechen keiner Frau ihre Handlungsfähigkeit von vornherein ab, sie nehmen Frauen als moralische Subjekte ihrer Entscheidung immer ernst.

Für die Identitätsfindung generell stellen prozedurale Konzeptionen jedoch insofern ein Problem dar, als sie nur auf formaler Ebene Hinweise auf ein gelingendes oder glückliches Leben liefern. Was gutes Leben inhaltlich ausmacht, wird durch prozedurale Konzeptionen bewusst nicht geklärt, sondern dem privaten Bereich des/der Einzelnen überlassen. Lipkina konstatiert, dass die Frage nach dem Guten, von deren Beantwortung so viel für geglückte Identität abhängt, zunehmend ins Private abgedrängt werde. ${ }^{49}$ Alle religiösen Traditionen als Diskursgemeinschaften stellen jedoch die Frage nach dem Guten und verknüpfen die Vorstellung von Autonomie mit Fragen des guten Lebens. Rein prozedurale Verfahrensregelungen genügen ihnen nicht.

Das Problem, das sich für die religiöse Identität von Frauen, die sich einer religiösen Tradition wie dem Christentum oder dem Islam zugehörig fühlen und gleichzeitig autonom im Sinne Pauer-Studers leben wollen, besteht nun in zweierlei Hinsicht: Erstens gibt eine rein prozedurale Konzeption keinen Hinweis darauf, welche Lebensform nun wirklich zu einem geglückten und sinnerfüllten Leben führen kann. Sie mag im Hinblick auf den mühsamen Prozess der eigenen Identitätsfindung zwar manchmal als befreiend erlebt werden, insofern sie keinerlei Vorgaben macht und die Authentizität und Unabhängigkeit des eigenen Weges gerade gegenüber traditionellen Vorgaben und/oder Autoritäten betont. Gerade aber weil sie keine Vorgaben kennt und alle Lebensformen als gleich gültig nebeneinander stellt, liefert sie auch wenig Orientierung auf dem Weg zum guten und geglückten Leben eines Selbst. Eine prozedurale Konzeption von personaler Autonomie bietet kaum Hinweise im Sinn einer moralischen Landkarte, wohin der Weg denn gehen soll. Vielleicht ist genau deswegen Identitätsbildung in der Postmoderne so schwierig geworden bzw. die Suche nach der eigenen Identität eines der großen Themen unserer Zeit.

Die Alternative kann dementsprechend nur in einer substantiellen Konzeption und damit einer inhaltlich gefüllten Vorstellung von personaler Autonomie liegen, doch ergeben sich hier gleich mehrere Probleme, die an dieser Stelle nur

48 Der Test der Authentizität und der prozeduralen Unabhängigkeit sorgt dennoch für eine notwendige Differenzierung, wenn er z. B. zur Klärung beiträgt, dass junge Frauen, die als angehende Bräute von IS-Terroristen angeworben werden, schlichtweg mit gezielten Fehlinformationen getäuscht worden sind, daher also als nicht autonom bezeichnet werden können.

49 Vgl. Lipkina 2016, S. 201. 
angedeutet werden können: Die heiligen Schriften aller religiösen Traditionen enthalten nicht nur Kritik an überkommenen Moralvorstellungen und emanzipatorisches Potential wie es sich in der Begegnung zwischen Jesus und der samaritanischen Frau am Jakobsbrunnen entfaltet. Sie sind oft genug Kinder ihrer Zeit. Dementsprechend transportieren sie ein patriarchales Ethos und eine moralische Bewertung diverser Lebensformen, die heutzutage nicht einfach unhinterfragt übernommen werden kann. Als konkretes Beispiel sei hier die alttestamentliche Vorstellung angeführt, dass für Frauen ein geglücktes Leben ohne Mutterschaft unvorstellbar ist und Kinderlosigkeit daher als heilloser Zustand bewertet wird, der über die individuelle Tragik hinaus noch dazu die Verheißung des Fortbestandes des Volkes Israels gefährdet, was den sozialen Druck auf kinderlose Frauen erhöht und durchaus nicht zu ihrer Befreiung beiträgt. Ein weiteres Beispiel, ebenfalls aus dem jüdisch-christlichen Erbe: Eine Pluralität von Lebensformen, wie sie die westlichen Gesellschaften heute kennen und die auch Formen homosexuellen Zusammenlebens beinhaltet, ist den Welten der Bibel fremd. Hier ist die traditionelle Familie heterosexueller Prägung die einzig denkbare Lebensform für das Zusammenleben der Geschlechter. Homosexuelle und lesbische Partnerschaften, wie sie heute ganz selbstverständlicher Teil der europäischen Gesellschaften geworden sind, sind schlicht undenkbar und überhaupt nicht im Blick. Der große Horizont guten Lebens enthält zwar Hoffnung auf das friedliche und erlöste Zusammenleben aller Menschen, doch steckt der Teufel im Detail moralischer Einzelnormen, die oft durchaus patriarchal gefärbt sind und ein Sippenethos enthalten, das mit der Pluralität von Lebensformen nicht ohne weiteres vereinbar ist. Alleine eine reflektierte Religion im Sinne einer hermeneutischen Arbeit an den Texten selbst schafft hier die notwendige Distanz, ohne den großen Horizont der Heilsverheißung für alle aus dem Blick zu verlieren.

Dass bei dieser genuin theologischen Arbeit Konflikte mit meist männlichen Autoritäten drohen, welche die Deutungshoheit über die Visionen guten Lebens beanspruchen und Vorstellungen von Geschlechtergerechtigkeit, die auf institutionell-strukturelle Veränderungen religiöser Traditionen abzielen und die Ansprüche von Frauen auf geteilte Leitungsverantwortung negieren oder sogar aktiv bekämpfen, liegt auf der Hand. Sie übersehen dabei das emanzipatorische Potential der eigenen jeweiligen religiösen Tradition, die den Weg zu mehr Geschlechtergerechtigkeit weisen könnte, wie ich in 2.3. darzustellen versucht habe. Die Erinnerung an die Erfahrung von Befreiung und die Verheißung eines erfüllten Lebens gerade an diejenigen, die nicht konform mit herkömmlichen moralischen Vorstellungen gehen, ist durchaus nicht überall willkommen, weil sie eine Relativierung und Hinterfragung traditioneller Lebensformen zur Folge haben kann. Entgegen der in 2.3. formulierten und biblisch gut fundierten Warnung einer Gleichsetzung von religiöser und moralischer Identität wird die Frage der Zugehörigkeit oder Nichtzugehörigkeit zu einer Religionsgemeinschaft aktuell durchaus anhand moralischer Bewertungen bestimmter Lebensformen 
Die religiöse Identität von Frauen im Spannungsfeld

vorgenommen bzw. unterliegen diejenigen, die den traditionellen Vorgaben nicht entsprechen, Mechanismen der Exklusion: Homosexuelle Menschen müssen auf eine sexuell aktive Partnerschaft verzichten, sonst können sie nicht Teil der katholischen Kirche sein; in islamischen Ländern werden sie häufig nach wie vor strafrechtlich verfolgt; Frauen/Männer, die sich jenseits zölibatärer Lebensformen in Ordensgemeinschaften bewusst für ein Leben ohne Partner/Partnerin entscheiden, finden in den christlichen Kirchen nur schwer einen Platz und auch im Islam ist eine solche Entscheidung rechtfertigungsbedürftig. Bereits eine prozedurale Vorstellung von personaler Autonomie mit ihrer Betonung von Authentizität und prozeduraler Unabhängigkeit kann eine Infragestellung traditioneller Lebensformen darstellen, wie sie durch Schrift und Tradition einer Religion mitbegründet, legitimiert und sanktioniert werden. Substantielle Autonomiekonzeptionen gehen jedoch noch einen Schritt weiter und lenken den Blick auf institutionelle und soziale Rahmenbedingungen, in denen normative Vorgaben verfestigt und weitertradiert werden, so dass sie am Ende fraglos internalisiert werden. Insbesondere patriarchale hierarchische Institutionen schaffen soziale Rahmenbedingungen, in denen Frauen kaum die Möglichkeit haben, tradierte Normen wirklich jemals in Frage zu stellen. Bei der Frage, ob eine Lebensform selbstbestimmt gewählt wurde, geht es daher in substantiellen Konzeptionen von personaler Autonomie weniger um die Entscheidung für eine bestimmte Lebensform selbst als um die Frage, ob eine echte Entscheidung unter den gegebenen Umständen überhaupt möglich ist. Damit stehen alle Lebensentwürfe von Frauen, die sich einer patriarchal geprägten religiösen Gemeinschaft zugehörig fühlen, sozusagen unter einem feministischen Generalverdacht: Leben sie wirklich selbstbestimmt? Oder folgen sie einfach tradierten Rollenmustern, die für beide Geschlechter unterschiedliche moralische Vorgaben vorsehen? Ist die angebliche Selbstbestimmung von Frauen, die sich einer religiösen Gemeinschaft zugehörig fühlen, letztlich und eigentlich eine internalisierte Fremdbestimmung religiöser Autoritäten und ihrer moralischen Vorschriften? Ist konsequenterweise dann erst in einer Institution mit real praktizierter Geschlechtergerechtigkeit auch echte Selbstbestimmung und Selbstermächtigung für Frauen möglich? Ein großer Teil religiöser Frauen müsste sich an dieser Stelle dann eingestehen, nicht wirklich selbstbestimmt zu sein, und tatsächlich: Ein substantiell gefasster Autonomiebegriff öffnet den Blick für den sozialen Kontext und nimmt das Gefüge von Institutionen kritisch unter die Lupe. Er stellt den Gerechtigkeitsanspruch für beide Geschlechter nicht nur ideell, sondern auch im Hinblick auf gleiche institutionelle Anerkennung für beide Geschlechter. ${ }^{50} \mathrm{Er}$ könnte genau deswegen zum Motor von Transformationsprozessen auch innerhalb religiöser Traditionen werden.

50 Andrea Günter hat die Verknüpfung von moralischer Identität mit Geschlechtergerechtigkeit anhand des Werks von Hannah Arendt aufgezeigt. Vgl. Günter 2004, S. 303-321. 


\subsection{Resümee: Identitätsdiskurse als Ringen um Autorität und Macht}

Warum stößt das Streben nach einer solch substantiell gefassten Autonomie bzw. die Forderung nach Geschlechtergerechtigkeit, wie sie aktuell im Christentum ${ }^{51}$ und auch - wenn auch in der Öffentlichkeit kaum wahrgenommen - im Islam erhoben wird $^{52}$, bei den meist männlichen Autoritäten bisher entweder auf wenig Resonanz oder auf offenen Widerstand? Vermutlich deshalb, weil diese überwiegend einem substanzmetaphysischen Paradigma verhaftet bleiben, welches die religiöse und moralische Identität von Frauen festschreibt, indem sie sie von einer männlichen Identität absetzt und dies mit der theologischen Schöpfungsordnung begründet. ${ }^{53}$ Postmoderne Identitätskonzeptionen, welche im Gegensatz dazu ein nomadisierendes und vagabundierendes Moment betonen und konsequenterweise die Vorstellung von einer religiösen und moralischen Identität entwickeln, die eben nicht mehr als starr, zeitenthoben und unabänderlich definiert werden kann, sondern als kontextualisiert und hybrid zu denken ist und sich in bestimmten Situationen immer wieder neu entwerfen muss, werden hier in ihrer Offenheit als Gefahr für den Machterhalt der eigenen Gruppe wahrgenommen. An der Schwelle, im Grenzbereich, ist die religiöse Identität von Frauen nicht nur in besonderem Maße offen für Gotteserfahrung. Sie ist auch hoch sensibel gegenüber Festschreibungen, welche über Zugehörigkeit und NichtZugehörigkeit entscheiden. Wenn also männliche religiöse Autoritäten kraft ihres Amtes und der Tradition entscheiden, wer den heiligen Raum betreten darf und wer nicht, dann müssen sie damit rechnen, dass vor allem theologisch gebildete Frauen, die sich des emanzipatorischen Potentials der eigenen Tradition bewusst sind und Religion nicht auf Moral reduzieren lassen, solche Entscheidungen in Frage stellen, möglicherweise nicht mittragen und den Binnenraum der eigenen Religion sogar verlassen - sei es in Resignation oder Zorn, sei es auch unter Einsatz von bewusster Respektlosigkeit. Arendt hat es folgendermaßen formuliert: „Autorität bedarf zu ihrer Erhaltung und Sicherung des Respekts entweder vor der Person oder vor dem Amt. Ihr gefährlichster Gegner ist nicht Feindschaft, sondern Verachtung, und was sie am sichersten unterminiert, ist das Lachen. ${ }^{\text {"54 }}$ Frauen, welche die Zugehörigkeit zu ihrer religiösen Tradition schätzen, dürften Verachtung und Lachen jedoch durchaus schwer fallen, insbesondere dort, wo

51 Konsequenz dieser Forderung ist das nun schon jahrzehntealte Ringen um die Ordination von Frauen in der katholischen Kirche. Zwar haben die Päpste der letzten Jahrzehnte Diskussionen darüber untersagt, doch ist damit das Thema nicht vom Tisch. 52 Vgl. Klausing 2014a, S. 94.

53 Nicht nur feministische Theologinnen haben gegen substanzmetaphysische Festschreibungen Protest eingelegt. Die gesamte Debatte um die Berechtigung der Kategorie Gender kann als Protest gegen essentialistische Identitätszuschreibungen gelesen werden. Vgl. Marschütz 2014.

54 Arendt 1990, S. 46. 
Autorität sich nicht allein auf das Amt gründet, sondern auf die persönliche Integrität eines Amtsinhabers. Eine feministische Theologie, die sich allein darin gefiele, Bild 1 und die dort abgebildeten männlichen Autoritäten zu belächeln und im Übrigen nicht mehr ernst zu nehmen bzw. feministische Parallelwelten zu errichten, reicht schon lange nicht mehr aus. Religiöse Frauen sind angesichts der Probleme dieser Welt genauso zu öffentlicher Leitungsverantwortung aufgefordert wie religiöse Männer. ${ }^{55}$ Bild 1 wird und muss sich verändern. Gerade theologisch gebildete Frauen sind nicht nur in die Pflicht genommen, von den männlichen Autoritäten ihrer religiösen Tradition institutionelle Geschlechtergerechtigkeit einzufordern, sondern die durchaus oft bequeme Rolle der Gehorsamen und der Genügsamen abzustreifen und sich nicht länger vor der Teilhabe an Macht und damit auch Verpflichtung und Last zu drücken. ${ }^{56}$

Identitätsdiskurse beinhalten das Ringen um die eigene Selbstbestimmung in Auseinandersetzung mit vorhandenen männlichen Autoritäten. Letztere berufen sich dabei allerdings gerne auf die Macht Gottes zur Legitimation bestehender Strukturen. Darauf hat im Prinzip schon Simone de Beauvoir hingewiesen, als sie den Zusammenhang zwischen der Fixierung einer aus der göttlichen Schöpfungsordnung abgeleiteten Identität der Frau und ihren entsprechenden moralischen Konsequenzen einerseits und der realen Machtpolitik in Religionen andererseits in ihrem Klassiker „Das andere Geschlecht“ offengelegt hat:

Es gibt eine große Rechtfertigung, einen höchsten Ausgleich, den die Gesellschaft der Frau immer zu bieten versucht hat: die Religion. Man braucht für die Frauen eine Religion, wie man eine für das Volk braucht, und aus genau den gleichen Gründen. Wer ein Geschlecht, eine Klasse zur Immanenz verurteilt, muss den Verurteilten notwendigerweise das Trugbild einer Transzendenz anbieten. Der Mann ist gut beraten, wenn er die Gesetze, die er macht, einem Gott zuschiebt. ${ }^{57}$

Die Behauptung einer spezifisch weiblichen und schöpfungstheologisch ein für alle Mal festgelegten Identität der Frau unter Berufung auf eine göttliche Schöpfungsordnung ist eindeutig Identitätspolitik, d. h. sie dient der Legitimation einer bestimmten Politik, die auf den Erhalt traditioneller institutioneller

55 Die hier vorliegende Untersuchung konzentriert sich fürs erste auf religiöse Frauen. Die Forschung der Zukunft sollte jedoch beide Geschlechter zum Gegenstand der Untersuchung machen. Wenn religiöse Frauen Bild 1 verändern, dann wird sich Bild 2 vermutlich mit verschieben. Auch insgesamt stellt sich die Frage nach dem Selbstbild von männlichen Autoritäten in religiösen Leitungsfunktionen und nach einer religiösen Identität von Männern. Sie bleiben von den derzeit stattfindenden Transformationsprozessen keineswegs unberührt und der Ruf nach Geschlechtergerechtigkeit wird von Verantwortlichen diverser religiöser Traditionen durchaus nicht nur ablehnend beurteilt.

56 Schaumberger 1988, S. 282-287.

57 de Beauvoir 2003, S. 773. 
Strukturen abzielt und Frauen dementsprechend von Leitungsfunktionen ausschließt. ${ }^{58}$

Doch religiöse Identitätsentwicklung - ob bei Männern oder Frauen - verläuft heute als offener Suchprozess. ${ }^{59}$ Die Rede von der Patchworkreligion und dementsprechend auch von einer religiösen Identität, die sich im Laufe des Lebens aus vielen Erfahrungen mit religiösen Traditionen und ihrer gegenseitigen Verwiesenheit in einer ,entangled history“ zusammensetzt, bringt diese Tatsache auf den Punkt. Kinder und Jugendliche begegnen im globalen Dorf religiösen Traditionen mit großer Offenheit. Sie suchen sich aus, was für sie in ihrer jeweiligen Lebenssituation hilfreich ist. Die Sehnsucht nach religiöser Erfahrung kann je nach Alter und Entwicklungsstand von der Herkunftsreligion gestillt werden, doch kann sie auch zum Ausbruch aus der vertrauten Religion des eigenen Kulturkreises führen. Die Frage, wie in den einzelnen religiösen Traditionen Geschlechtergerechtigkeit (nicht) praktiziert wird, dürfte ein entscheidender Faktor sein, dass gerade junge Frauen ihre Herkunftsreligion immer öfter bewusst verlassen und sich auf die Suche nach anderen religiösen Sinnsystemen begeben.

Wie die individuelle Entwicklung von Frauen verläuft, die auf der Suche nach der eigenen religiösen Identität sind, dürfte in Zukunft in noch höherem Maße als bisher von der Begegnung mit religiösen Autoritäten abhängen. ${ }^{60}$ Bild 1 wird sich auf lange Sicht ändern müssen, wenn religiöse Traditionen ihrem eigenen emanzipatorischen Potential treu bleiben und religiöse Frauen nicht zunehmend an alternative säkulare Anbieter verlieren wollen. Jedenfalls ist mit der Forderung nach substantieller Autonomie die Sehnsucht nach Geschlechtergerechtigkeit und ihrer praktischen Umsetzung nicht mehr aus der Welt zu schaffen, auch nicht mehr aus der Welt der religiösen Traditionen. Insbesondere theologisch gebildete Frauen werden immer lauter auf ihre Realisierung drängen.

Die religiöse Identität von Frauen, welche die Möglichkeit von personaler Autonomie als theologisch begründet erkannt hat, bezieht aus der Gotteserfahrung ihre Kraft im Ringen mit männlichen Autoritäten um institutionelle Geschlechtergerechtigkeit und Partizipation von Frauen in Leitungsfunktionen. Bei aller Fluidität und Offenheit: Die Schwellenidentität religiöser Frauen wirkt systemverändernd.

58 Dieser Zusammenhang war Gegenstand eines Symposiums in Wien 2016. Vgl. Internetquelle: Symposium Gender.

59 Vgl. Roebben 2011, S. 48-57.

60 Es ist ein Forschungsdesiderat an die kritische Männerforschung, die spezifisch männlich religiöse Identitätsentwicklung empirisch zu untersuchen. Die Tatsache der überwiegend männlichen Repräsentanz von Religion schafft hier meiner Vermutung nach andere Voraussetzungen als bei religiösen Frauen. 


\section{Literaturverzeichnis}

Arendt, Hannah: Macht und Gewalt. München ${ }^{7} 1990$.

Benhabib, Seyla: Selbst im Kontext. Gender Studies. Frankfurt am Main 1995.

Beauvoir, Simone de: Das andere Geschlecht. Sitte und Sexus der Frau. Hamburg 2003.

Bhabha, Homi K..: The Location of Culture. London / New York 1994.

Braidotti, Rosi: Nomadic Subjects. New York 1993.

Casanova, José: „Public Religions Revisited“, in: de Vries, Hent (Hg.): Religion. Beyond a Concept. New York 2008, S. 101-119.

Danz, Christian: „Religiöse Identität und gesellschaftliche Integration. Zur Funktion von Religion in gesellschaftlichen Inklusions- und Exklusionsprozessen“, in: Rothgangel, Martin / Aslan, Ednan / Jäggle, Martin (Hgs.): Religion und Gemeinschaft. Göttingen 2013, S. 33-47.

Dworkin, Gerald: The Theory and Practice of Autonomy. Cambridge 1988.

Dworkin, Gerald: „The Concept of Autonomy“, in: Christman, John (Hg.): The Inner Citadel. Essays on individual autonomy. New York/Oxford 1989, S. 54-62.

Günter, Andrea: „Die weibliche Hoffnung der Welt und die Suche nach Rückbindung: Zum Verhältnis von Politik, Religion und Frauenbewegung“, in: Pfleiderer, Georg / Stegemann, Ekkehard W. (Hgs.): Politische Religion. Geschichte und Gegenwart eines Problemfeldes. Zürich 2004, S. 303-321.

Günter, Andrea: „Gerechtigkeit durch Gleichheit/Differenz oder Gleichheit/Differenz durch Gerechtigkeit“", in: Riedl, Anna Maria / Kroll, Anna / Krause, Felix / Hartlieb, Michael (Hgs.): Gender - Autonomie - Identität. Beobachtungen, Konzepte, sozialethische Reflexionen (Forum Sozialethik 15). Münster 2015, S. 83-95.

Habermann, Ruth: „Das Evangelium nach Johannes. Orte der Frauen“, in: Schottroff, Luise / Wacker, Marie-Theres (Hgs.): Kompendium Feministische Bibelauslegung. Gütersloh ${ }^{3} 2007$, S. 530-533.

Habermas, Jürgen: Glauben und Wissen. Friedenspreis des Deutschen Buchhandels 2001. Frankfurt 2001.

Habermas, Jürgen: Zwischen Naturalismus und Religion. Philosophische Aufsätze. Frankfurt am Main 2005.

Habermas, Jürgen: „,Das Politische“ - der vernünftige Sinn eines zweifelhaften Erbstücks der Politischen Theologie“, in: Mendieta, Eduardo / Van Antwerpen, Jonathan (Hgs.): Religion und Öffentlichkeit. Berlin 2012, S. 28-52.

Heimbach-Steins, Marianne: ,... nicht mehr Mann und Frau“. Sozialethische Studien zu Geschlechterverhältnis und Geschlechtergerechtigkeit. Regensburg 2009.

Heimbach-Steins, Marianne / Wielandt, Rotraud / Zintl, Reinhard (Hgs.): Religiöse Identität(en) und gemeinsame Religionsfreiheit. Eine Herausforderung pluraler Gesellschaften. Würzburg 2006, S. 9-24.

Heller, Birgit: „Geschlechtergerechtigkeit in den Religionen? Systematisch-religionswissenschaftliche Perspektiven“, in: Elsas, Christoph / Franke, Edith / Staudhartinger, Angela (Hgs.): Geschlechtergerechtigkeit: Herausforderung der Religionen. Göttingen 2014, S. 29-44.

Heller, Birgit / Heller, Andreas: Spiritualität und Spiritual Care. Orientierungen und Impulse. Bern 2014.

Hervieu-Leger, Daniele: Religion as a chain of memory. Cambridge 2000. 
Joan Wallach, Scott: „Secularism“, in: The politics of the veil. Princeton 2007, S. 90-123. Klausing, Kathrin: „Muslimische Positionen zu Feminismus - Begriffe, Bewegungen, Methoden“, in: Franke, Yvonne / Mozygemba, Kati / Pöge, Kathleen / Ritter, Bettina / Venohr, Dagmar (Hgs.): Feminismen heute. Positionen in Theorie und Praxis. Bielefeld 2014a, S. 89-99.

Klausing, Kathrin: „Weibliche islamische Gelehrte als Gegenstand muslimischen Interesses“, in: Elsas, Christoph / Franke, Edith / Staudhartinger, Angela (Hgs.): Geschlechtergerechtigkeit: Herausforderung der Religionen. Göttingen 2014b, S. 241251.

Kluge, Friedrich: Etymologisches Wörterbuch der deutschen Sprache. Berlin ${ }^{22} 1989$.

Lipkina, Julia: Identität als Voraussetzung für Bildung. Eine qualitative Studie zu Bildungserfahrungen als Frage nach der Ermöglichung von Identität in schulischen und außerschulischen Kontexten. Berlin 2016.

Mandry, Christof: Ethische Identität und christlicher Glaube. Theologische Ethik im Spannungsfeld von Theologie und Philosophie. Mainz 2002.

Meyer-Wilmes, Hedwig: Rebellion auf der Grenze. Ortsbestimmung feministischer Theologie. Freiburg im Breisgau 1990.

Mieth, Dietmar: Moral und Erfahrung. Grundlagen einer theologisch-ethischen Hermeneutik. (Studien zur theologischen Ethik 2). Freiburg / Wien 1999.

Nedelsky, Jennifer: „Reconceiving Autonomy: Sources, Thoughts and Possibilities“, in: Yale Journal of Law and Feminism (7) 1989, S. 7-36.

Niamh, Reilly: „Rethinking the interplay of feminism and secularism in a neo-secular age", in: feminist review (97) 2011, S. 5-31.

Nunner-Winkler, Gertrud: „Der Mythos von den zwei Moralen“, in: Deutsche Zeitschrift für Philosophie (42) 1994, S. 237-254.

Oshana, Marina A.L.: Personal Autonomy in Society. Burlington 2006.

Oshana, Marina A.L.: „Is social-relational personal autonomy a plausible ideal“, in: Oshana, Marina A.L. (ed.): Personal Autonomy and Social Oppression. Philosophical perspectives. New York, 2015, S. 3-24.

Pauer-Studer, Herlinde: „Ethik und Geschlechterdifferenz“, in: Nida-Rümelin, Julian (Hg.): Angewandte Ethik. Die Bereichsethiken und ihre theoretische Fundierung. Ein Handbuch. Stuttgart 1996, S. 87-136.

Pauer-Studer, Herlinde: Autonom leben. Reflexionen über Freiheit und Gleichheit. Frankfurt am Main 2000.

Polak, Regina / Reiss, Wolfram (Hgs.): Religion im Wandel. Transformation religiöser Gemeinschaften in Europa durch Migration - Interdisziplinäre Disziplinen. Göttingen 2014.

Pollack, Detlef: „Religiöser Wandel in Ost- und Westeuropa: Soziologische Beschreibungen und Erklärungen“, in: Appel, Kurt / Guanzini, Isabella / Walser, Angelika (Hgs.): Europa mit oder ohne Religion? Der Beitrag der Religion zum gegenwärtigen und künftigen Europa. Göttingen 2014, S. 93-120.

Reilly, Niamh: ,rethinking the interplay of feminism and secularism in a neo-secular age“, in: feminist review (97) 2011, S. 5-31.

Rettenbacher, Sigrid: „Interreligiöse Theologie - postkolonial gelesen“, in: Bernhardt, Reinhold / Schmidt-Leukel, Perry (Hgs.): Interreligiöse Theologie. Chancen und Probleme. Zürich 2013, S. 67-111. 
Roebben, Bert: Religionspädagogik der Hoffnung. Grundlinien religiöser Bildung in der Spätmoderne. Berlin 2011.

Schaumberger, Christine / Schottroff, Luise: Schuld und Macht. Studien zu einer feministischen Befreiungstheologie. München 1988.

Scott, Joan W.: „Sexularism“, in: RSCAS Distinguished Lectures (01) 2009, verfügbar unter: http://cadmus.eui.eu/bitstream/handle/1814/11553/RSCAS_DL_2009_01.pdf? sequence $=1$ [24.04.2017].

Sommer, Regina: „Aspekte eines feministisch-theologischen Konzepts von Identität“, in: Gössmann, Elisabeth: Wörterbuch der Feministischen Theologie. Gütersloh 2002, S. 294-296.

Walser, Angelika: Die Autonomie von Frauen in bioethischen Konfliktfeldern zu Beginn des Lebens als Herausforderung für die Theologische Ethik. Habilitationsschrift. Wien 2013, verfügbar in der Universitätsbibliothek Wien, siehe: http://ubdata.univie.ac.at/ AC11592846 [24.04.2017].

Walser, Angelika: „Freundschaft“ im interdisziplinären Dialog: Perspektiven aus Philosophie, Theologie, Sozialwissenschaften und Gender Studies (Salzburger Theologische Studien 59). Innsbruck 2017.

Warriner, Jennifer: „Gender oppression and weak substantive theories of autonomy“, in: Oshana, Marina A.L. (ed.): Personal Autonomy and Social Oppression. Philosophical perspectives. New York, 2015, S. 25-47.

Zulehner, Paul M. / Steinmair-Pösel, Petra: Typisch Frau? Wie Frauen leben und glauben. Linz 2011.

\section{Internetquellen}

„Frauen als Diakone: Papst rührt an Tabu“, in: ORF, verfügbar unter: http://religion.orf. at/stories/2774131/ [24.04.2017].

„Frauenhetz“, verfügbar unter: http://frauenhetz.jetzt/ [24.04.2017].

Girau Pieck, Gabrielle / Hafner Al Jabaji, Amira / Lenzin, Rifa'at / Pruschy, Eva / Rudolf, Heidi / Strahm, Doris / Traitler, Reinhild: „Rabbinerinnen, Kantorinnen, Imaminnen, Muftis, Pfarrerinnen, Bischöfinnen, Kirchenrätinnen ... Leitungsfunktionen von Frauen im Judentum, im Christentum und im Islam“, in: Interreligiöser Think-Tank 2011, verfügbar unter: http://www.interrelthinktank.ch/index.php/texte/texte-des-in terreligioesen-think-tank-2/item/21-rabbinerinnen-kantorinnen-imaminnen-muftispfarrerinnen-bischoefinnen-kirchenraetinnen [24.04.2017].

IRPA, verfügbar unter: http://www.irpa.ac.at [24.04.2017].

Lutheranworld, https://www.lutheranworld.org/sites/default/files/DTPW-WICAS_Gen der_Justice-DE.pdf [30.3.2017].

Marschütz, Gerhard: „Wachstumspotential für die eigene Lehre“, in: Herder Korrespondenzen 2014, verfügbar unter: https:/www.herder-korrespondenz.de/heftarchiv/ 68-jahrgang-2014/heft-9-2014/zur-kritik-an-der-vermeintlichen-gender-ideologiewachstumspotenzial-fuer-die-eigene-lehre [24.04.2017].

Studien des Schweizer Interreligiösen Think-Tanks 2011, verfügbar unter: http://www. interrelthinktank.ch/archivos/ITT_Studie_2011_web.pdf [30.9.2016]. 
„Symposium ,Gender““, verfügbar unter: https://st-theoethik-ktf.univie.ac.at/newsevents/events/symposium-gender/ [21.04.2017].

\section{Bildnachweise}

Bild 1: „Kirchen gemeinsam zum Schutz der Nahost-Christen verpflichtet“, in: Erzdiözese Wien, verfügbar unter: https://www.erzdioezese-wien.at/site/home/nachrichten/ article/39822.html [24.04.2017].

Bild 2: Caritas Wien, https:/www.caritas-wien.at/fileadmin/storage/wien/hilfe-angebote/ zusammenleben/nachbarschaft/jamie-ministry-food.jpg [24.04.2017].

Univ.Prof.in Dr.in Angelika Walser, Moraltheologie und Spirituelle Theologie, Fachbereich Praktische Theologie, Universität Salzburg, Universitätsplatz 1, 5020 Salzburg, Austria, angelika.walser@sbg.ac.at

Citation: Walser, Angelika: „Die religiöse Identität von Frauen im Spannungsfeld von Zugehörigkeit, Autorität und Autonomie“, in: Heller, Birgit (ed.): Religion, Transformation and Gender. (J-RaT 2017 / 2), pp. 27-54.

Datum der Publikation: 02.10.2017 